\section{Imaging Cerebral Malaria with a Susceptibility-Weighted MR Sequence}

Cerebral malaria is a severe complication seen in approximately $2 \%$ of patients with acute Plasmodium falciparum infection. Characteristic diffuse petechial hemorrhages that were previously only seen by gross pathologic or microscopic examination, and virtually undetectable by conventional imaging modalities, were well demonstrated in a patient with cerebral malaria, with use of susceptibility-weighted imaging (SWI). This case highlights the usefulness of SWI imaging to accelerate the diagnosis of cerebral malaria.

A 31-year-old woman was seen in the emergency department within 1 week of returning from a trip to the Philippines, complaining of cyclic fever and chills, headache, nausea, and vomiting. She admitted to inconsistently taking her malaria prophylaxis medication while out of the country. Her initial admission blood smear result was positive for $P$ falciparum. A head CT scan was obtained; results were unremarkable. She was admitted to the intensive care unit, where she required intubation for respiratory failure. Head MR imaging was obtained 3 days after admission and demonstrated diffuse petechial hemorrhage throughout the gray-white matter junction, corpus callosum, and internal capsules of both hemispheres on SWI as well as edema in the posterior limbs of the bilateral internal capsules on fluid-attenuated inversion recovery imaging (Fig 1). The patient was extubated on hospital day 10 and was discharged home on day 12.
Follow-up MR imaging at 6 weeks continued to demonstrate diffuse small hemorrhages with some interval improvement and resolution of the previously noted edema (Fig 2).

Cerebral malaria is a rare but potentially fatal complication of infection with $P$ falciparum. Diffuse petechial hemorrhage occurs in these cases when cerebral capillaries and small veins are occluded by infected erythrocytes. Although the mortality rate of cerebral malaria has been estimated between $20 \%$ and $50 \%$, those patients who survive often have a full recovery with no long-term sequelae.

Imaging findings in cerebral malaria have been limited in the past. Significant hemorrhage or infarction with resultant edema is required for CT detection. Millan et $\mathrm{al}^{1}$ described MR imaging findings in cerebral malaria by using conventional T1-weighted and T2-weighted imaging to demonstrate hemorrhage and infarction in a single case, and Cordoliani et $\mathrm{al}^{2}$ reported a series of 12 patients with cerebral malaria who received MR imaging, only 3 of whom demonstrated any findings suggestive of a pathologic process on conventional T1 and T2 sequences. The SWI sequence described by Tong et $\mathrm{al}^{3}$ is much more sensitive in the detection of small hemorrhages than is conventional MR imaging, including standard 2D gradient-echo $\mathrm{T} 2{ }^{*}$ sequences. This high spatial-resolution technique is extremely sensitive to susceptibility changes. SWI has been shown to be helpful in the detection of small hemorrhages in various conditions including diffuse axonal injury, coagulopathy, neoplasms, and neurodegenerative disorders. ${ }^{4}$
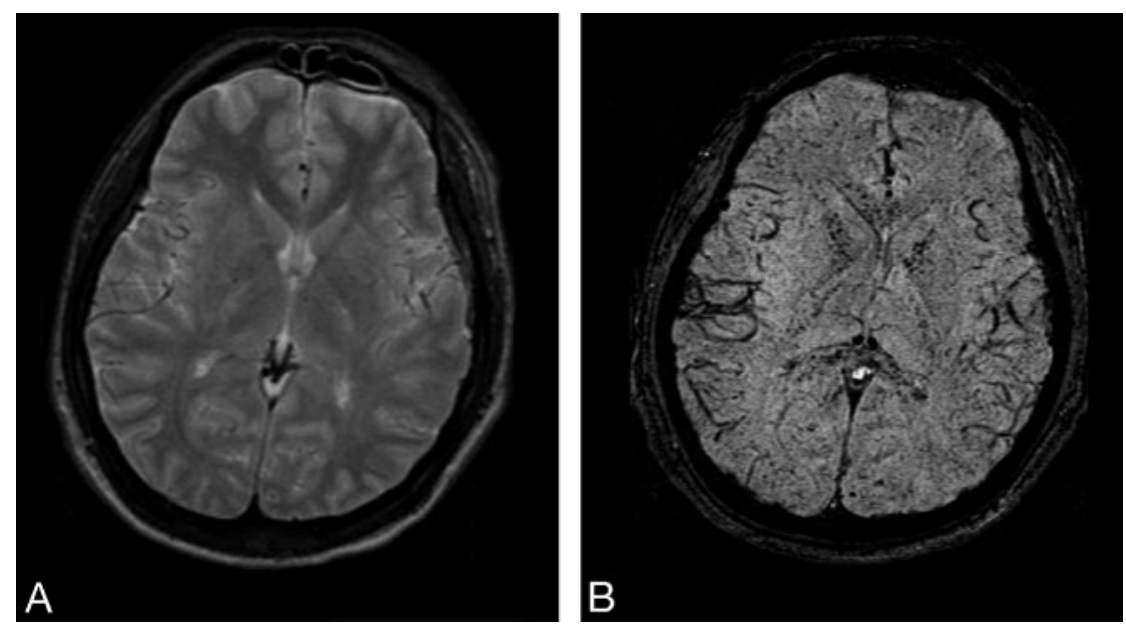

Fig 1. Admission MR images ( $A$ and $B)$ : T2 and SWI. Diffuse petechial hemorrhages present throughout the brain (particularly the gray-white junction, corpus callosum, and internal capsule) are seen only on the SWI sequence.
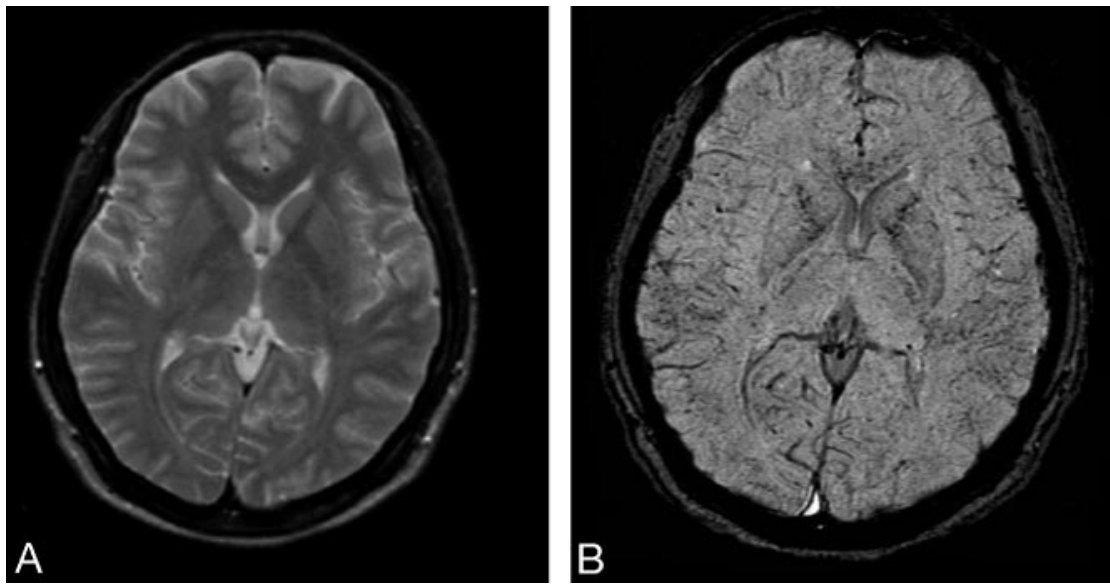

Fig 2. Six-week follow-up study $(A$ and $B)$ : T2 and SWI. SWI reveals residual hemorrhages, though there has been some interval improvement. 
Imaging findings with use of this sequence seem to correlate much more closely with the expected pathologic findings of diffuse petechial hemorrhage. In the future, SWI may play a significant role in the detection of small hemorrhagic lesions that are not detectable by conventional MR images or CT scan.

\section{References}

1. Millan J, San Millan J, Munoz M, et al. CNS complications in acute malaria: MR findings. AJNR Am J Neuroradiol 1993;14:493-94

2. Cordoliani YS, Sarrazin JL, Felten D, et al. MR of cerebral malaria. AJNR Am J Neuroradiol 1998;19:871-74

3. Tong KA, Ashwal S, Holshouser BA, et al. Hemorrhagic shearing lesions in children and adolescents with posttraumatic diffuse axonal injury: improved detection and initial results. Radiology 2003;227:332

4. Tong KA, Ashwal S, Obenaus A, et al. Susceptibility-weighted MR imaging: a review of clinical applications in children. AJNR Am J Neuroradiol 2008;29:9-17.

J.P. Nickerson

Fletcher Allen Healthcare

University of Vermont

Department of Radiology

Burlington, $V t$

K.A. Tong

Department of Neuroradiology

R. Raghavan

Department of Pathology

Loma Linda University Medical Center

Loma Linda, Calif

DOI 10.3174/ajnr.A1568 